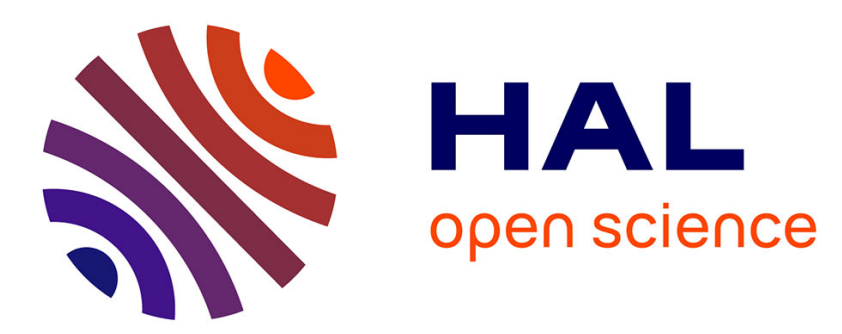

\title{
Etude de la compétition pollinique chez le sulla (Hedysarum coronarium L., Légumineuse-Papilionacée)
}

Ali Chriki, Daniel Combes, Mohamed Marrakchi

\section{To cite this version:}

Ali Chriki, Daniel Combes, Mohamed Marrakchi. Etude de la compétition pollinique chez le sulla (Hedysarum coronarium L., Légumineuse-Papilionacée). Agronomie, 1984, 4 (2), pp.155-159. hal00884622

\section{HAL Id: hal-00884622 \\ https://hal.science/hal-00884622}

Submitted on 1 Jan 1984

HAL is a multi-disciplinary open access archive for the deposit and dissemination of scientific research documents, whether they are published or not. The documents may come from teaching and research institutions in France or abroad, or from public or private research centers.
L'archive ouverte pluridisciplinaire HAL, est destinée au dépôt et à la diffusion de documents scientifiques de niveau recherche, publiés ou non, émanant des établissements d'enseignement et de recherche français ou étrangers, des laboratoires publics ou privés. 


\title{
Etude de la compétition pollinique chez le sulla (Hedy- sarum coronarium L., Légumineuse-Papilionacée)
}

\author{
Ali CHRIKI, Daniel COMBES $\left(^{*}\right) \&$ Mohamed MARRAKCHI \\ Laboratoire de Génétique, Faculté des Sciences, Campus Universitaire Tunis, Tunisie \\ (*) Université de Pau et des Pays de l'Adour, avenue Philippon F 64000 Pau
}

RÉSUMÉ

Par l'observation de la couleur des fleurs dans des descendances de pollinisation naturelle et de croisements chez Hedysarum coronarium L., on montre que l'allopollen est tellement favorisé par rapport à l'autopollen que 90 p. 100 des graines sont issues d'une fécondation croisée.

L'étude de la compétition pollinique chez cette espèce montre également que les gènes de coloration a et $b$ ne semblent pas avoir une liaison particulière avec un gène gamétophytique mâlc.

Mots clés additionnels : Autogamie, allogamie, pollinisation naturelle, pollinisation artificielle, pollinisation pluripaternelle, gène de coloration, compétition gamétique.

The character «presence-absence » of flower anthocyanins was used as a genetic marker in the study of pollen competition in sulla (Hedysarum coronarium L.). This species was shown to be preferentially allogamous. The rate of allogamy obtained under natural and artificial pollinisation was close to $90 \%$.

The use of different sorts of pollen in simple crosses without castration and in multipaternal pollination experiments showed that the two flower colour genes, $a$ and $b$, were not apparently linked to a male gamctophyte gene.

Additional key words: Autogamy, allogamy, natural pollination, artificial pollination, multipaternal pollination, flower colour genes, gametic competition.

\section{INTRODUCTION}

Hedysarum coronarium L., ou sulla, est une légumineuse à vocation fourragère qui fait l'objet de plusicurs travaux de recherches depuis quelques années. Ces travaux sont axés essenticllement sur la détermination de ses qualités fourragères, l'étude de sa reproduction et l'analyse de sa variabilité morphologique (GRIMALDl, 1961 ; COMBES et al., 1975 ; FigIER et al., 1978).

Les résultats mentionnés jusqu'à présent montrent que cette espèce à tendance allogame présente une valeur nutritive équivalente à celle de la luzerne et du trèfle violet mais nettement supćricure à celle du foin de prairic. Les pcuplements naturels de cette espèce sont utilisés par les agriculteurs tunisiens soit pour le pâturage direct des ovins et des bovins, soit sous forme de fourrage fauché. Cependant, l'extension de sa culture était freinée en particulier du fait que les génotypes utilisés étaient essenticllement producteurs de rameaux plagiotropes.

En vue d'améliorer cette espèce pour son utilisation comme plante de pâturage en Tunisie, une analyse de la morphogenèse en liaison avec la structure génétique ainsi qu'une étude détaillée des populations naturelles pour en apprécier la variabilité paraissaient donc nécessaires tant du point de vue fondamental que du point de vue appliqué. Pour réaliser un tel travail avec succès, il paraît nécessaire d'étudier, dans une première étape, le mode de reproduction chez cette espèce. Il semble que le sulla soit une plante préférentiellement allogame (GRIMALDI, 1961) sans pour autant écarter la possibilité de l'autofécondation.

Cette tendance à l'allogamic laisse supposer des interactions de nature biochimique entre le sporophyte et son propre pollen favorisant ainsi la fécondation croisée. Mais, jusqu'alors, on ne dispose pas d'une estimation précise du taux d'allogamic chez cette espèce. Pour mieux évaluer ce taux, nous avons essayé d'estimer les taux de fécondation croisée en pollinisation naturelle (par les abeilles) et en pollinisation artificiclle (manuelle). Pour ce faire, nous avons utilisé des marqueurs génétiques, liés à la coloration florale chez cette espèce, que nous avons nous-mêmes mis en évidence (CHRIKI et al., 1982b). 


\section{MATÉRIEL ET MÉTHODES}

Les plantes utilisées dans cette étude sont soit homozygotes pour 2 marqueurs de coloration florale et de génotype $\mathrm{AABB}, \mathrm{aaBB}$ ou $\mathrm{AAbb}$, soit simples hétérozygotes de génotype $\mathrm{AABb}$. L'hérédité des 2 paires d'allèles de la coloration des fleurs, $\mathrm{Aa}$ et $\mathrm{Bb}$, a été étudiéc précédemment (CHRIKI et al., 1982b). Les allèles récessifs, a et b, suppriment la coloration anthocyanique dans la plante entičre et ne semblent pas agir sur la coloration jaune des anthères.

\section{A. Taux d'allogamie en pollinisation naturelle}

Des plantes homozygotes à fleurs blanches de génotype $\mathrm{AAbb}$ et des plantes hétérozygotes à fleurs rouges de génotype $\mathrm{AABb}$ ont été placées en plein champ d' $H$. coronarium à fleurs rouges où la fréquence des gènes récessifs impliqués dans le déterminisme de l'absence de pigmentation anthocyanique est supposée nulle.

Le taux d'allogamie est donné par la proportion de plantes à fleurs colorécs dans la descendance des plantes à fleurs blanches; alors que lc taux d'autogamic cst fourni par la proportion de plantes à fleurs blanches dans la même descendance.

Nous désignons par pollen «sauvage» le pollen de génotype $\mathrm{AB}$ et par pollen «muté » celui de génotype $\mathrm{Ab}$ ou aB.

Pour étudier la compétition entre les 2 types d'allopollen, "muté » et «sauvage», nous avons adopté la méthode de mélange de grains de pollen déjà utilisée par PlonkA et al. (1968).

Dans cette expérience, nous avons pollinisé, après castration, une forme maternelle à fleurs blanches, de génotype $\mathrm{AAbb}$ ou aaBB, avec un mélange en proportions approximativement égales de pollen provenant de 2 formes paternelles différentes, l'une à fleurs blanches, de génotype $A A b b$ ou aaBB, et l'autre à fleurs rouges, de génotype AABB. Nous avons ensuite analysé du point de vue phénotypique la descendance obtenue par pollinisation pluripaternellc.

\section{B. Taux d'allogamie en pollinisation artificielle}

Nous avons essayé d'effectuer des croisements sans castration qui se rapprochent autant que possible des croisements naturels, afin que le stigmate reçoive à la fois de l'auto et de l'allopollen.

Les génotypes des formes maternelles et parternelles ont été choisis de façon à pouvoir étudier les relations entre les types de pollen qui se distinguent par les allèles de la coloration qu'ils apportent et par leur éventuel effet sur les interactions «style-pollen».

\section{RÉSULTATS}

\section{A. Taux d'allogamie en pollinisation naturelle}

Comme c'est le cas de la plupart des légumincuses, la pollinisation croisée chez $H$. coronarium cst assuréc par les insectes dont les abeilles (Apis mellifica, notamment), les bourdons et les mégachiles. La pollinisation anémophile ne semble pas jouer un rôle important chez cette espèce.

\section{Taux d'allogamie chez les plantes à fleurs blanches}

La descendance des plantes à fleurs blanches (tabl. 1) pollinisées librement se compose de 83 plantes à fleurs
TABLEAU 1

Taux d'allogamie en pollinisation naturelle chez Hedysarum coronarium $L$.

Rate of allogamy for natural pollination in Hedysarum coronarium $L$.

\begin{tabular}{|c|c|c|c|c|}
\hline \multirow[t]{2}{*}{$\begin{array}{c}\text { Phénotype } \\
\text { des } \\
\text { parents }\end{array}$} & \multirow[t]{2}{*}{$\begin{array}{l}\text { Géno- } \\
\text { types }\end{array}$} & \multicolumn{2}{|c|}{$\begin{array}{l}\text { Composition } \\
\text { phénotypique } \\
\text { de la } \\
\text { descendance } \\
\text { en PL }\end{array}$} & \multirow[t]{2}{*}{$\begin{array}{c}\text { Taux } \\
\text { d'allogamie }\end{array}$} \\
\hline & & $\mathbf{P}$ & NP & \\
\hline NP & $\mathrm{AAbb}$ & 83 & 10 & 0,90 \\
\hline $\mathrm{P}$ & $\mathrm{AABb}$ & 160 & 5 & 0,88 \\
\hline
\end{tabular}

P : pigmenté ; NP : non pigmenté ; $\mathrm{PL}$ : pollinisation libre.

rouges et 10 plantes à fleurs blanches. Le taux d'allogamie, $\mathrm{t}$, qui correspond à la proportion des plantes pigmentées dans la descendance est de 0,90 .

Cette valeur de $\mathrm{t}$ est, a priori, sous-estimée étant donné que la couleur blanche des fleurs réfléchit la lumière et que les radiations de courte longueur d'onde sont répulsives pour les butineuses. D'autre part, les effets de l'homogamie ct de la préférence des abeilles pour un phénotype donné (phénomène de fixité) pourraient modifier la valeur du taux d'allogamie chez les mutants à fleurs blanches. Pour réduire l'influence de ces facteurs, nous avons déterminé le taux d'allogamie en utilisant des plantes pigmentées hétérozygotes.

\section{Taux d'allogamie chez les plantes pigmentées hétérozy- gotes}

La descendance des plantes hétérozygotes à fleurs rouges pollinisées librement (tabl. 1) se compose de 160 plantes pigmentécs et 5 plantes non pigmentées.

En désignant par $t$ le taux d'allogamie et en supposant que les plantes non pigmentées ne peuvent provenir que d'autofécondation, sur les 165 descendants, 165 (1-t) proviennent d'autofécondation et, parmi ceux-ci, 1/4, soit $\frac{165}{4}(1-t)$ sont à fleurs blanches. Par conséquent, $\frac{165(1-\mathrm{t})}{4}=5$ d'où $\mathrm{t}=0,88$.

Cette valeur de $t$ ne semble pas s'ćcarter significativement de cellc obtenue précédemment pour les plantes à fleurs blanches pollinisées librement (tabl. 2). Les effets de l'homogamic et de la préférence des abeilles pour un phénotype donné seraicnt négligcables.

\section{B. Compétition entre allopollens de génotypes différents}

Pour chercher s'il existe une relation entre les gènes de la coloration a et b et le gamétophyte mâle, c'est-à-dire le tube pollinique, des expériences de fécondation utilisant des mélanges de pollen de génotypes différents ont ćté réalisécs (tabl. 3). Des séries de croisements ont été effectućs faisant entrer en compétition le pollen « sauvage » $A B$, soit avec lc pollen "muté » $\mathrm{Ab}$, soit avec le pollen $\mathrm{aB}$.

Dans le cas où une compétition cxiste entre les 2 types d'allopollen, «muté » et «sauvage», celle-ci pourra avoir une répercussion sur la composition phénotypique des descendances issues de pollinisation pluripaternelle. 


\section{TABLEAU 2}

Comparaison des taux d'allogamie en pollinisation naturelle des plantes pigmentées hétérozygotes et des plantes non pigmentées. Comparison of rates of allogamy for natural pollination of heterozygous red flowering plants and acyanic mutants.

\begin{tabular}{|c|c|c|c|}
\hline \multirow[t]{2}{*}{ Plantes issues de } & \multicolumn{3}{|c|}{ Pollinisation naturelle des plantes } \\
\hline & $\begin{array}{c}\text { Non } \\
\text { pigmentées }\end{array}$ & Pigmentécs & Total \\
\hline Autofécondation & 10 & $20\left(^{*}\right)$ & 30 \\
\hline Fécondation croisée & 83 & 145 & 228 \\
\hline Total & 93 & 165 & 258 \\
\hline
\end{tabular}

Analyse statistique :

$\mathrm{X}_{\mathrm{c}}^{2}=0,016 ; \quad 0,50<\mathrm{P}<0,9$, non significatif.

$\mathrm{X}_{\mathrm{c}}^{2}=\mathrm{X}^{2}$ corrigé de Yates.

$\left.{ }^{*}\right)$ Valeur estimée en appliquant la formule $n=165(1-t)$. Dans cette formule $\mathrm{t}$ désigne le taux d'allogamie chez les plantes hétérozygotes.

\section{TABLEAU 3}

Composition phénotypique de descendances de pollinisation pluripaternelle.

Phenotypic composition of progenies from multipaternal pollination.

\begin{tabular}{|c|c|c|c|}
\hline \multirow[t]{2}{*}{ Croiscment } & \multicolumn{3}{|c|}{$\begin{array}{c}\text { Composition phénotypique } \\
\text { de la descendance }\end{array}$} \\
\hline & Pigmenté & $\begin{array}{c}\text { Non } \\
\text { pigmenté }\end{array}$ & Total \\
\hline \multirow{2}{*}{$\begin{array}{l}q \mathrm{AAbb} \times \delta \delta \mathrm{AAbb}, \\
\mathrm{AABB} \\
q \mathrm{aaBB} \times \delta \delta \\
\mathrm{AABB}\end{array}$} & 13 & 10 & 23 \\
\hline & 25 & 21 & 46 \\
\hline Total & 38 & 31 & 69 \\
\hline
\end{tabular}

Analyse statistique :

- $\mathrm{X}^{2}$ d'homogénéité $=0,74 ; 0,30<\mathrm{P}<0,50$, non significatif.

- $\mathrm{X}_{\mathrm{c}}^{2}$ (ségrégation $\left.1: 1\right)=0,52 ; 0,30<\mathrm{P}<0,50$, non significatif.

Les descendances de ces 2 types de croisements étant statistiquement homogènes $\left(\mathrm{X}^{2}=0,74 ; 0,30<\mathrm{P}<0,50\right.$, tabl. 3), il est possible de les cumuler en une seule descendance globalc qui se compose de 38 plantes pigmentées et 31 plantes non pigmentées. Dans cette descendance, les proportions des plantes pigmentées et non pigmentées, respectivement égales à 0,55 et 0,45 , ne s'écartent pas significativement des proportions 0,5: 0,5 attendues, en absence de compétition entre pollens de génotypes différents $\left(X_{c}^{2}=0,52 ; 0,30<P<0,5\right.$, tabl. 3$)$.

Les expériences de pollinisation avec des mélanges de pollens porteurs de gènes de coloration différents n'impliquent pas une disproportion qui pourrait se produire en cas de compétition entre les tubes polliniques au niveau du sporophyte femellc.

Il semble donc que les gènes de coloration a et b n'ont pas d'action significative sur la vitesse de croissance des tubes polliniques dans le style. Mais ceci n'exclut pas une action éventuelle de ces gènes sur le gamétophyte femelle (le sac embryonnaire en voie de formation) et sur le zygote et son devenir. Nous avons remarqué, en effet, une germination moins vigoureuse des graines et une moins bonne fertilité des gousses chez les plantes dépourvues de pigments anthocyaniques.

Notons que cette chute de vigueur et de fertilité pourrait être également un effet dépressif de la consanguinité. Ceci peut être confirmé en comparant, dans la descendance en autofécondation d'un hétérozygote, les plantes colorées et les plantes à fleurs blanches.

\section{Taux d'allogamie en pollinisation artificielle}

Selon le génotype des différents parents utilisés, 3 cas seront envisagés (tabl. 4).

\section{Compétition entre allopollen "sauvage» et autopollen "muté »}

La descendance du croisement, sans castration, $\mathrm{AAbb} \times \mathrm{AABB}$ se compose de 39 plantes à flcurs rouges et de 4 plantes à fleurs blanches (tabl. $4 a$ ). A partir de ces données, nous avons déterminé le taux d'allogamie, soit $\mathrm{t}=0,907$.

Dans le croisement sans castration $\mathrm{AAbb} \times \mathrm{AABB}$, le stigmate reçoit de l'allopollen $\mathrm{AB}$ et de l'autopollen $\mathrm{Ab}$. Cette situation nous rappelle celle de la pollinisation naturelle des plantes à fleurs blanches placées en plein champ d' $H$. coronarium à fleurs rouges.

Il est donc possible de comparer, pour les plantes à fleurs blanches, le taux d'allogamie en pollinisation artificiclle au taux d'allogamie en pollinisation naturelle (tabl. 1). On trouve une différence non significative à 5 p. 100 $\left(X^{2}=0,002 ; P>0,9\right.$, tabl. 5).

Il semble donc que les croisements manuels (à l'aide d'une lame de verre) donnent des résultats analogues à ceux fournis par les croisements naturels réalisés par les insectes.

\section{TABLEAU 4}

Taux d'allogamie en pollinisation artificielle. Rate of allogamy for artificial pollination.

\begin{tabular}{|c|c|c|c|}
\hline \multirow{2}{*}{$\begin{array}{c}\text { Croisement } \\
\text { sans castration }\end{array}$} & \multicolumn{2}{|c|}{$\begin{array}{c}\text { Composition } \\
\text { de la descendance }\end{array}$} & \multirow{2}{*}{$\begin{array}{c}\text { Taux } \\
\text { d'allogamie }\end{array}$} \\
\hline & Pigmenté & $\begin{array}{c}\text { Non } \\
\text { pigmenté }\end{array}$ & \\
\hline (a) $q \mathrm{AAbb} \times \delta \mathrm{AABB}$ & 39 & 4 & 0,907 \\
\hline (b) ( ) aaBB $\times \delta \mathrm{AAbb}$ & 36 & 4 & 0,900 \\
\hline (c) $q \mathrm{AAbb} \times \delta \mathrm{AABb}$ & 9 & 11 & 0,900 \\
\hline
\end{tabular}

\section{TABLEAU 5}

Comparaison des taux d'allogamie en pollinisations naturelle et artificielle.

Comparison of the rates of allogamy for natural and artificial pollination.

\begin{tabular}{lccc}
\hline \hline Plantes issue de & \multicolumn{3}{c}{ Plantes obtenues en } \\
& PL & PA & Total \\
\hline Autofécondation & 10 & 4 & 14 \\
Fécondation croisée & $\frac{83}{93}$ & $\frac{39}{43}$ & $\frac{122}{136}$ \\
Total & 93 & &
\end{tabular}

$\mathrm{PL}$ : pollinisation libre; $\mathrm{PA}$ : pollinisation artificielle.

Analyse statistique : $X_{\mathrm{e}}^{2}=0,002 ; P>0,90$, non significatif. 
Dans un croisement naturel, le stigmate semble recevoir beaucoup plus d'autopollen que d'allopollen. Chez la luzerne par exemple, le stigmate reçoit de l'autopollen et de l'allopollen approximativement dans les proportions respectives 4 : 1 (DEMARLY, 1963), alors que dans un croisement manuel, le stigrnate reçoit de l'autopollen et de l'allopollen en proportions sensiblement égales; le résultat des 2 types de croisements, naturel et manuel est néanmoins le même (tabl. 5). Par conséquent, la fécondation de l'oosphère par un type donné de pollen ne dépend pas de sa densité mais vraisemblablement des interactions biochimiques entre le style et les divers pollens et de la compétition entre l'auto et l'allopollen.

\section{Compétition entre allo et autopollens "mutés"}

Le croisement, sans castration, aaBB $\times$ AAbb, donne une descendancie qui se compose de 36 plantes pigmentées et 4 plantes non pigmentées (tabl, $4 b$ ). Ces résultats fournissent un taux d'allogamic, $\mathrm{t}=0,90$.

Les taux d'allogamie obtenus respectivement avec un allopollen "sauvage " $\mathrm{AB}$ et un allopollen "muté " $\mathrm{Ab}$ (tabl. $4 a$ et $4 b$ ) ne sont pas significativement différents à 5 p. $100\left(\mathrm{X}_{\mathrm{c}}^{2}=0,070 ; 0,50<\mathrm{P}<0,90\right.$, tabl. 6).

Il semble donc que les différents gènes de la pigmentation apportés par le pollen n'ont aucun effet sur le taux d'allogamie en situation de compétition du type "alloautopollen ». Céci confirme le fait que les gènes impliqués dans le déterminisme de la pigmentation anthocyanique chez $H$. coronarium ne sont pas liés à un gène gamétophytique mâle.

En outre, dans l'ćtude génétique de la pigmentation anthocyanique chez $H$. coronarium (CHRIKI et al., 1982a) nous n'avons pas noté, en $\mathrm{F}_{2}$, des écarts significatifs par rapport aux proportions mendéliennes. Notons que ces écarts peuvent être produits par un gène gamétophytique (Plonka, 1968, 1971; Singh \& Dulieu, 1979). C'est un argument supplementaire en faveur de l'hypothèse de la non-liaison des gènes $a$ et $b$ à un gène gamétophytique chez cette espèce.

3. Compétition entre autopollen "muté " et 2 types d'allopollen, "muté » et "sauvage"

Sans castrer les fleurs, nous avons réalisé le croisement $\mathrm{AAbb} \times \mathrm{AABb}$ qui a fourni 9 plantes pigmentées et 11 plantes non pigmentécs (tabl. $4 c$ ). De cc résultat, nous avons déduit le taux d'allogamic, $\mathrm{t}=0,90$.

Les proportions des plantes pigmentées et non pigmen-

\section{TABLEAU 6}

Comparaison des taux d'allogamie respectivement obtenus avec un allopollen $A B$ et un allopollen $A b$.

Comparison of the rates of allogamy respectively obtained with $A B$ allopollen and Ab allopollen.

\begin{tabular}{lccc}
\hline \hline Plantes issues de & \multicolumn{3}{c}{ Plantes obtenucs } \\
& $\mathrm{AB}$ & $\mathrm{Ab}$ & Total \\
\hline Autofécondation & 4 & 4 & 8 \\
Fécondation croiséc & $\frac{39}{43}$ & $\frac{36}{40}$ & $\frac{75}{83}$ \\
Total & 43 & & \\
\hline
\end{tabular}

Analyse statistique : $\mathrm{X}_{\llcorner}^{2}=0,070 ; 0,50<\mathrm{P}<0,90$, non significatif. tées observées dans la descendance de ce croisement sans castration ne s'écartent pas significativement des proportions $1: 1$, attendues dans un véritable croisement récurrent avec castration $\left(\mathrm{X}_{\mathrm{c}}^{2}=0,05 ; 0,50<\mathrm{P}<0,90\right)$.

Un tel résultat est très important sur le plan pratique, puisqu'il nous permet, tout en négligeant la fraction autofécondation, de réaliser des croisements sans avoir recours à unc castration préalable des fleurs, opération techniquement assez délicate.

Etant donné que le croisement récurrent $\mathrm{AAbb} \times \mathrm{AABb}$ donne une descendance normale $1: 1$, sans qu'il y ait un déficit marqué de récessifs ou de dominants, les 2 types d'allopollen $\mathrm{AB}$ et $\mathrm{Ab}$ doivent présenter le même pouvoir compétitif vis-à-vis de l'autopollen $\mathrm{Ab}$. Il semble donc que la croissance des tubes polliniques de l'allopollen, « muté » ou " sauvage ", est tellement favorisée par rapport à celle de l'autopollen que 90 p. 100 des graines sont issues d'une fécondation croiséc.

\section{DISCUSSION ET CONCLUSION}

L'utilisation de marqueurs génétiques «présenceabsence " de pigmentation anthocyanique nous a permis de préciser quelques aspects de la biologie de la reproduction chez $H$. coronarium.

Les résultats obtenus sont en accord avec ceux des travaux de Grimaldi (1961), COMBES et al. (1975), Figier et al. (1978) et confirment que le régime de reproduction de cette espèce est préférenticllement allogame sans pour autant écarter la possibilité d'autofécondation.

Cette tendance à l'allogamie chez le sulla semble être le résultat de divers mécanismes liés à la biologie florale de la plante. En particulier, nous avons constaté (résultats non publiés) qu'au sein d'une fleur donnée, la maturité du pollen précède la réceptivité (protandric).

Dans les croisements mettant en compétition l'auto et l'allopollen, nous avons montré que l'allopollen est avantagé et assurc la fécondation dans 90 p. 100 des cas. Les taux d'allogamie obtenus en pollinisation naturelle ne s'écartent pas significativement de ceux obtenus en pollinisation artificielle.

Un taux d'autogamie de 10 p. 100 n'influe pas significativement sur les résultats des croisements sans castration. Ceci nous permet d'effectuer des croisements sans avoir recours à unc castration préalablc. Dans un croisement avec castration, l'ablation mécanique des anthères est non sculcment délicate mais elle risque généralement de blesser les organes femelles. C'est la raison pour laquelle, il est matériellement difficile d'obtenir des descendances à cffectifs relativement élcvés.

Chez la luzerne, plante allogame, la croissance des tubes polliniques de l'allopollen est tellement favoriséc par rapport à celle de l'autopollen que 95 p. 100 des graines sont issues d'une fécondation croiséc (DEMARLY, 1963).

Chez les plantes allogames, il existe généralement un locus d'incompatibilité muni de toute une série d'allèles $S$. Exceptionnellement, il y en a 2 chez les graminées et les solanacées avec la supposition d'unc origine par duplication de l'autrc locus (selon LUNDQvisT, 1965, cité par PLONKA, 1971).

La famille des légumineuses à laquelle appartient le sulla, contient de nombreuses espèces diploïdes possédant un système d'incompatibilité de type gamćtophytique (LEwIS, 1979). Si ce système détermine à lui scul l'allogamie, la réaction du pollen scra toujours celle de l'allèle d'incompatibilité qu’il portc. 
Chez $H$. coronarium, la pseudocompatibilité observéc peut être due à la présence d'allèles d'autofertilité au locus S, comme c'est le cas de Trifolium hybridum (TOWNSEND, 1965). Elle peut également provenir de la présence de gènes modificateurs. Dans ce cas, l'incompatibilité serait placée sous un contrôle polygénique en même temps que le gène $S$. L'incompatibilité du trèfle violet peut être modifiée par les ségrégations de gènes modificateurs ou par une sélection (selon DENWARD, 1963, cité par DATTEE, 1971).

Les résultats obtenus ne permettent pas de préciser le système d'incompatibilité chez $H$. coronarium. Mais, l'hypothèse d'un système d'incompatibilité sous un contrôle polygénique, en dehors du gène majeur $\mathrm{S}$, semble être la plus vraisemblable pour expliquer la pseudocompatibilité observée chez cette espèce. Dans tous les cas, la compétition pollinique jouc un rôle non négligeable dans la réalisation de l'allogamie.

Lorsqu'un caractère morphologique est lié, dans le grain de pollen, à l'un des gènes agissant sur le tube pollinique, on observe des déviations de disjonction pour ce caractère (Plonka et al., 1968 ; PlonkA, 1971 ; Singh \& Dulieu, 1979). Au cours de l'étude de la compétition pollinique, nous n'avons pas noté une variation du taux d'allogamic ni une disproportion dans les descendances en rapport avec les gènes de la coloration portés par le pollen.

On pense que chez $H$. coronarium, les gènes de la coloration $\mathrm{a}$ et $\mathrm{b}$ ne seraient pas liés à un gène gamétophytique mâle.
On peut rapprocher ce cas de mutation aux gènes $\mathrm{a}^{\mathrm{d}}$ et $\mathrm{1}^{\mathrm{m}}$ chez le lin cultivé (PlonKA, 1971), intervenant respectivement dans la suppression des pigments bleus dans le limbe des pétales, les anthères et les stigmates ainsi que dans la production d'un pigment mauve dans les pétales. Ces 2 gènes ne paraissent pas liés à un gène gamétophytique mâle.

En défavorisant le sporophyte, c'est-à-dire la plante, les gènes $a$ et $b$ peuvent être rapprochés du gène $p^{b}$ responsable de la suppression de toute couleur anthocyanique chez le lin cultivé (PlonKA, 1971). En effet ce gène, en même temps qu'il désavantage le sporophyte et défavorise le gamétophyte femelle, n'a pas d'action sur le gamétophyte mâle.

Il serait cependant nécessaire de compléter les résultats obtenus dans l'analyse de la compétition pollinique soit par une étude in vivo de la vitesse de croissance des tubes polliniques d'allo et autopollen (DESPREZ \& BANNEROT, 1980), soit par une étude in vitro du comportement des 2 types de pollen mis en présence l'un de l'autre, en dehors de toute interaction style-pollen (DEBRAND et al., 1979). On peut également utiliser la technique d'étude en semi-in situ des relations style pollen décrite chez la luzerne (ABDELKEFI, 1980).

Reçu le 7 mars 1983. Accepté le 16 septembre 1983.

\section{REMERCIEMENTS}

Ce travail a profité de l'aide de l'ACCT, du CNRS et du FIS.

\section{RÉFÉRENCES BIBLIOGRAPHIQUES}

Abdelkefi A., 1980. Technique d'étude en semi in situ des relations stylc-pollen chez la luzerne (Medicago sativa L.). Ann. Amélior. Plantes, 30, 271-284.

Chriki A., Combes D., Marrakchi M., 1982a. Mise en ćvidence de deux types de mutants acyaniques chez l'Hedysarum coronarium $\mathrm{L}$. C. R. Acad. Sci., Paris, 294, 739-742.

Chriki A., Combes D., Marrakchi M., 1982b. Hérédité et analyse chromatographique de la pigmentation des fleurs chez l'espèce Hedysarum coronarium L. Agronomie, 2 (10), 915-923.

Combes D., Espagnac H., Figier J., 1975. Etude de populations naturelles d'Hedysarum coronarium L. du Nord de la Tunisie. Bull. Soc. Hist. Nat. Afr. Nord, 66, 107-122.

Dattee Y., 1971. Analyse quantitative de l'auto et de l'interfertilité chez quelques familles de luzerne (Medicago sativa L.). Thèse $3^{\circ}$ cycle, Faculté des Sciences de Paris, 111 p.

Debrand M., Cornuet J. M., Dattee Y., Guy P., 1979. Etude in vitro de la compétition pollinique chez quelques génotypes de luzerne (Medicago sativa L.). Ann. Amélior. Plantes, 29 (1), 63-77.

Demarly Y., 1963. Génétique des tétraplö̈des et amélioration des plantes. Ann. Amélior. Plantes, 13, 307-408.

Desprez F., Bannerot H., 1980. A study of pollen tube growth in witloof chicory. Proceedings Eucarpia Meeting on leafy vegetables. Littlehampton, 11-14 mars 1980. Edited by Glasshousc Crops Research Institute, 47-52.
Figier J., Espagnac H., Combes D., Francillon G., 1978. Mise en évidence de types morphologiques dans les populations naturclles de l'H. coronarium de Tunisie par analyse multivariable. Rev. Gen. Bot., 85, 21-62.

Grimaldi A., 1961. Observazioni e ricerche morfobiologiche sopra la Sulla (Hedysarum coronarium L.). Ann. Fac. Agr., 16, 3-28. Lewis D., 1979. Sexual Incompatibility in Plants. Studies in Biology $N^{\circ}$ 110. Edward Arnold Publ. Itd., London, 60 p.

Plonka F., 1971. La compétition pollinique chez le lin cultivé. Ann. Amélior. Plantes, 21, 179-220.

Plonka F., Dubois J., Arnoux J., 1968. Les fécondations avec des mélanges de pollen comme moyen d'étude de la sćlection gamétophytique dans les styles du lin cultivé. Ann. Amélior. Plantes, 18 (3), 261-271.

Singh I. S., Dulieu H. L., 1979. Compétition pollinique chez des mutants nucléaires de tabac induits par le rayonnement gamma. Ann. Amélior. Plantes, 29 (3), 253-266.

Townsend C. E., 1965. Sclf compatibility studies with diploid alsike clover, Trifolium hybridum L. (I) Frequency of self-compatible plants in diverse populations and inheritance of a self-compatibility factor Sf. Crop Sci., 5, 358-360. 